\title{
Loop Subdivision Surface based Progressive Interpolation
}

\author{
Fuhua (Frank) Cheng ${ }^{a}$, Fengtao Fan $^{a}$, Shuhua Lai ${ }^{b}$ \\ Conglin Huang ${ }^{a}$, Jiaxi Wang ${ }^{a}$, Junhai Yong ${ }^{c}$ \\ ${ }^{a}$ Department of Computer Science, University of Kentucky, Lexington, KY 40506, USA \\ ${ }^{b}$ Department of Mathematics and Computer Science, Virginia State University, Petersburg, VA 23806, USA \\ ${ }^{c}$ School of Software, Tsinghua University, Beijing 100084, P.R. China
}

\begin{abstract}
A new method for constructing interpolating Loop subdivision surfaces is presented. The new method is an extension of the progressive interpolation technique for B-splines. Given a triangular mesh $M$, the idea is to iteratively upgrade the vertices of $M$ to generate a new control mesh $\bar{M}$ such that limit surface of $\bar{M}$ would interpolate $M$. It can be shown that the iterative process is convergent for Loop subdivision surfaces. Hence, the method is well-defined. The new method has the advantages of both a local method and a global method, i.e., it can handle meshes of any size and any topology while generating smooth interpolating subdivision surfaces that faithfully resemble the shape of the given meshes. The meshes considered here can be open or closed.
\end{abstract}

\section{Introduction}

Subdivision surfaces are becoming popular in many areas such as animation, geometric modeling and games because of their capability in representing any shape with only one surface. A subdivision surface is generated by repeatedly refining a control mesh to get a limit surface. Hence, a subdivision surface is determined by the way the control mesh is refined, i.e., the subdivision scheme. A subdivision scheme is called an interpolating scheme if the limit surface interpolates the given control mesh. Otherwise, it is called an approximating scheme. Popular subdivision schemes such as Catmull-Clark scheme [2], Doo-Sabin scheme [1], and Loop scheme [8] are approximating schemes while the Butterfly scheme [6], the improved Butterfly scheme [9] and the Kobbelt scheme [18] are interpolating schemes.

An interpolating subdivision scheme generates new vertices by performing local affine combinations on nearby vertices. This approach is simple and easy to implement. Because of its local property, it can handle meshes with a large number of vertices. However, since no vertex is ever moved once it is computed, any distortion in the early stage of the subdivision will persist. This makes interpolating subdivision schemes very sensitive to irregularity in the given mesh. In addition, it is difficult for this approach to interpolate normals or derivatives.

On the other hand, even though subdivision surfaces generated by approximating subdivision schemes do not interpolate their control meshes, it is possible to use this approach to generate a subdivision surface to interpolate the vertices of a given mesh. One method, called global optimization, does the work by building a global linear system with some fairness constraints to avoid undesired undulations $[3,4]$. The solution to the global linear system is a control mesh whose limit surface interpolates the vertices of the given mesh. Because of its global property, this method generates smooth interpolating subdivision surfaces that resemble the shape of the given meshes well. But, for the same reason, it is difficult for this method to handle meshes with a large number of vertices.

To avoid the computational cost of solving a large system of linear equations, several other methods have been proposed. A two-phase subdivision method that works for meshes of any size was presented by Zheng and Cai for Catmull-Clark scheme [5]. A method proposed by Lai and Cheng 
[15] for Catmull-Clark subdivision scheme avoids the need of solving a system of linear equations by utilizing the concept of similarity in the construction process. Litke, Levin and Schöder avoid the need of solving a system of linear equation by quasi-interpolating the given mesh [7]. However, a method that has the advantages of both a local method and a global method is not available yet.

In this paper a new method for constructing a smooth Loop subdivision surface that interpolates the vertices of a given triangular mesh is presented. The new method is an extension of the progressive interpolation technique for B-splines [10, 12, 13]. The idea is to iteratively upgrade the locations of the given mesh vertices until a control mesh whose limit surface interpolates the given mesh is obtained. It can be proved that the iterative interpolation process is convergent for Loop subdivision surfaces. Hence, the method is well-defined for Loop subdivision surfaces. The limit of the iterative interpolation process has the form of a global method. But the control points of the limit surface can be computed using a local approach. Therefore, the new technique enjoys the advantages of both a local method and a global method, i.e., it can handle meshes of any size and any topology while generating smooth interpolating subdivision surfaces that faithfully resemble the shape of the given meshes. The meshes considered here can be open or closed.

The remaining part of the paper is arranged as follows. In Section 2, we present the concept of progressive interpolation for Loop subdivision surfaces on closed meshes. In section 3 , we prove the convergence of this iterative interpolation process. Extension of this technique to open meshes is considered in Section 4. Implementation issues and test results are presented in Section 5. Concluding remarks are given in Section 6.

\section{Progressive Interpolation using Loop Subdivision Surfaces for Closed Meshes}

The concept of Loop subdivision surface based progressive interpolation for closed meshes can be described as follows.

Given a closed 3D triangular mesh $M=M^{0}$. To interpolate the vertices of $M^{0}$ with a Loop subdivision surface, one needs to find a closed control mesh $\bar{M}$ whose Loop surface passes through all the vertices of $M^{0}$. Instead of finding the relationship between the vertices of $\bar{M}$ and the vertices of $M^{0}$ directly, we use an iterative process to do the job.

First, we consider the Loop surface $\mathbf{S}^{0}$ of $M^{0}$. For each vertex $\mathbf{V}^{0}$ of $M^{0}$, we compute the distance between this vertex and its limit point $\mathbf{V}_{\infty}^{0}$ on $\mathbf{S}^{0}$,

$$
\mathbf{D}^{0}=\mathbf{V}^{0}-\mathbf{V}_{\infty}^{0}
$$

and add this distance to $\mathbf{V}^{0}$ to get a new vertex called $\mathbf{V}^{1}$ as follows:

$$
\mathbf{V}^{1}=\mathbf{V}^{0}+\mathbf{D}^{0}
$$

The set of all the new vertices is called $M^{1}$. We then consider the Loop surface $\mathbf{S}^{1}$ of $M^{1}$ and repeat the same process.

In general, if $\mathbf{V}^{k}$ is the new location of $\mathbf{V}^{0}$ after $k$ iterations of the above process and $M^{k}$ is the set of all the new $\mathbf{V}^{k}$ 's, then we consider the Loop surface $\mathbf{S}^{k}$ of $M^{k}$. We first compute the distance between $\mathbf{V}^{0}$ and the limit point $\mathbf{V}_{\infty}^{k}$ of $\mathbf{V}^{k}$ on $\mathbf{S}^{k}$

$$
\mathbf{D}^{k}=\mathbf{V}^{0}-\mathbf{V}_{\infty}^{k}
$$

We then add this distance to $\mathbf{V}^{k}$ to get $\mathbf{V}^{k+1}$ as follows:

$$
\mathbf{V}^{k+1}=\mathbf{V}^{k}+\mathbf{D}^{k}
$$

The set of new vertices is called $M^{k+1}$.

This process generates a sequence of control meshes $M^{k}$ and a sequence of corresponding Loop surfaces $\mathbf{S}^{k}$. $\mathbf{S}^{k}$ converges to an interpolating surface of $M^{0}$ if the distance between $\mathbf{S}^{k}$ and $M^{0}$ 
converges to zero. Therefore the key task here is to prove that $\mathbf{D}^{k}$ converges to zero when $k$ tends to infinity. This will be done in the next section.

Note that for each iteration in the above process, the main cost is the computation of the limit point $\mathbf{V}_{\infty}^{k}$ of $\mathbf{V}^{k}$ on $\mathbf{S}^{k}$. For a Loop surface, the limit point of a control vertex $\mathbf{V}$ with valence $n$ can be calculated as follows:

$$
\mathbf{V}_{\infty}=\beta_{n} \mathbf{V}+\left(1-\beta_{n}\right) \mathbf{Q}
$$

where

$$
\beta_{n}=\frac{3}{11-8 \times\left(\frac{3}{8}+\left(\frac{3}{8}+\frac{1}{4} \cos \frac{2 \pi}{n}\right)^{2}\right)}
$$

and

$$
\mathbf{Q}=\frac{1}{n} \sum_{i=1}^{n} \mathbf{Q}_{i}
$$

$\mathbf{Q}_{i}$ are adjacent vertices of $\mathbf{V}$. This computation involves nearby vertices only. Hence the progressive interpolation process is a local method and, consequently, can handle meshes of any size.

Another point that should be pointed out is, even though this is an iterative process, one does not have to repeat each step strictly. By finding out when the distance between $M^{0}$ and $\mathbf{S}^{k}$ would be smaller than the given tolerance, one can go directly from $M^{0}$ to $M^{k}$, skipping the testing steps in between.

\section{Convergence of the Iterative Interpolation Process for Closed Meshes}

The proof needs a fact about the eigenvalues of the product of positive definite matrices. This fact is presented in the following lemma.

Lemma 1 Eigenvalues of the product of positive definite matrices are positive.

The proof of Lemma 1 follows immediately from the fact that if $P$ and $Q$ are square matrices of the same dimension, then $P Q$ and $Q P$ have the same eigenvalues (see, e.g., [16], p.14).

To prove the convergence of the iterative interpolation process for Loop subdivision surfaces, note that at the $(k+1)$ st step, the difference $\mathbf{D}^{k+1}$ can be written as:

$$
\begin{aligned}
\mathbf{D}^{k+1} & =\mathbf{V}^{0}-\mathbf{V}_{\infty}^{k+1} \\
& =\mathbf{V}^{0}-\left(\beta_{n} \mathbf{V}^{k+1}+\left(1-\beta_{n}\right) \mathbf{Q}^{k+1}\right)
\end{aligned}
$$

where $\mathbf{Q}^{k+1}$ is the average of the $n$ adjacent vertices of $\mathbf{V}^{k+1}$

$$
\mathbf{Q}^{k+1}=\frac{1}{n} \sum_{i=1}^{n} \mathbf{Q}_{i}^{k+1}
$$

By applying (2) to $\mathbf{V}^{k+1}$ and each $\mathbf{Q}_{i}^{k+1}$,

$$
\mathbf{Q}_{i}^{k+1}=\mathbf{Q}_{i}^{k}+\mathbf{D}_{\mathbf{Q}_{i}^{k}}^{k}
$$

we get

$$
\begin{aligned}
\mathbf{D}^{k+1}= & \mathbf{V}^{0}-\left(\beta_{n} \mathbf{V}^{k}+\left(1-\beta_{n}\right) \mathbf{Q}^{k}\right) \\
& -\left(\beta_{n} \mathbf{D}^{k}+\frac{1-\beta_{n}}{n} \sum_{i=1}^{n} \mathbf{D}_{\mathbf{Q}_{i}}^{k}\right) \\
= & \mathbf{D}^{k}-\left(\beta_{n} \mathbf{D}^{k}+\frac{1-\beta_{n}}{n} \sum_{i=1}^{n} \mathbf{D}_{\mathbf{Q}_{i}}^{k}\right)
\end{aligned}
$$


where $\mathbf{Q}^{k}$ is the average of the $n$ adjacent vertices of $\mathbf{V}^{k}$. In matrix form, we have

$$
\left[\mathbf{D}_{1}^{k+1}, \mathbf{D}_{2}^{k+1}, \ldots, \mathbf{D}_{m}^{k+1}\right]^{T}=(I-B)\left[\begin{array}{c}
\mathbf{D}_{1}^{k} \\
\mathbf{D}_{2}^{k} \\
\vdots \\
\mathbf{D}_{m}^{k}
\end{array}\right]=(I-B)^{k+1}\left[\begin{array}{c}
\mathbf{D}_{1}^{0} \\
\mathbf{D}_{2}^{0} \\
\vdots \\
\mathbf{D}_{m}^{0}
\end{array}\right]
$$

where $m$ is the number of vertices in the given matrix, $I$ is an identity matrix and $B$ is a matrix of the following form:

$$
\left(\begin{array}{cccc}
\beta_{n_{1}} & \cdots & \frac{1-\beta_{n_{1}}}{n_{1}} & \cdots \\
\vdots & \ddots & & \\
\frac{1-\beta_{n_{i}}}{n_{i}} & \cdots & \beta_{n_{i}} & \cdots \\
\vdots & & & \beta_{n_{m}}
\end{array}\right)
$$

The matrix $B$ has the following properties:

(1) $b_{i j} \geq 0$, and $\sum_{j=1}^{n} b_{i j}=1$ (hence, $\|B\|_{\infty}=1$ );

(2) There are $n_{i}+1$ positive elements in the $i$-th row, and the positive elements in each row are equal except the element on the diagonal line.

(3) If $b_{i j}=0$, then $b_{j i}=0$;

Properties (1) and (2) follow immediately from the formula of $\mathbf{D}^{k+1}$ in eq. (3). Property (3) is true because if a vertex $\mathbf{V}_{i}$ is an adjacent vertex to $\mathbf{V}_{j}$ then $\mathbf{V}_{j}$ is obviously an adjacent vertex to $\mathbf{V}_{i}$. Due to these properties, we can write the matrix $B$ as

$$
B=D S
$$

where $D$ is a diagonal matrix

$$
\mathbf{D}=\left(\begin{array}{cccc}
\frac{1-\beta_{n_{1}}}{n_{1}} & 0 & \cdots & 0 \\
0 & \frac{1-\beta_{n_{2}}}{n_{2}} & \cdots & 0 \\
\vdots & & \ddots & \\
0 & & & \frac{1-\beta_{n_{m}}}{n_{m}}
\end{array}\right)
$$

and $S$ is a symmetric matrix of the following form:

$$
\mathbf{S}=\left(\begin{array}{cccc}
\frac{n_{1} \beta_{n_{1}}}{1-\beta_{n_{1}}} & \ldots & 1 & \ldots \\
\vdots & \ddots & & \\
1 & \ldots & \frac{n_{i} \beta_{n_{i}}}{1-\beta_{n_{i}}} & \ldots \\
\vdots & & & \frac{n_{m} \beta_{n_{m}}}{1-\beta_{n_{m}}}
\end{array}\right)
$$

$D$ is obviously positive definite. We will show that the matrix $S$ is also positive definite, a key point in the convergence proof.

Theorem 1 The matrix $S$ is positive definite.

Proof: To prove $S$ is positive definite, we have to show the quadric form

$$
f\left(x_{1}, x_{2}, \ldots, x_{m}\right)=\mathbf{X}^{T} S \mathbf{X}
$$

is positive for any non-zero $\mathbf{X}=\left(x_{1}, x_{2}, \ldots, x_{m}\right)^{T}$. 
Note that if vertices $\mathbf{V}_{i}$ and $\mathbf{V}_{j}$ are the endpoints of an edge $e_{i j}$ in the mesh, then $s_{i j}=s_{j i}=1$ in the matrix $S$. Hence, it is easy to see that

$$
\begin{aligned}
f\left(x_{1}, x_{2}, \ldots, x_{n}\right)= & \sum_{e_{i j}} 2 x_{i} x_{j} \\
& +\sum_{i=1}^{m} \frac{n_{i} \beta_{n_{i}}}{1-\beta_{n_{i}}} x_{i}^{2}
\end{aligned}
$$

where $e_{i j}$ in the first term ranges through all edges of the given mesh. On the other hand, if we use $f_{i j r}$ to represent a face with vertices $\mathbf{V}_{i}, \mathbf{V}_{j}$ and $\mathbf{V}_{r}$ in the mesh, then since an edge in a closed triangular mesh is shared by exactly two faces, the following relationship holds:

$$
\begin{aligned}
\sum_{f_{i j r}}\left(x_{i}\right. & \left.+x_{j}+x_{r}\right)^{2} \\
= & \sum_{e_{i j}} 4 x_{i} x_{j}+\sum_{i=1}^{m} n_{i} x_{i}^{2}
\end{aligned}
$$

where $f_{i j r}$ on the left hand side ranges through all faces of the given mesh. The last term in the above equation follows from the fact that a vertex with valence $n$ is shared by $n$ faces of the mesh. Hence, $f\left(x_{1}, x_{2}, \ldots, x_{n}\right)$ can be espressed as

$$
\begin{aligned}
f\left(x_{1}, x_{2}, \ldots, x_{n}\right)= & \sum_{f_{i j r}} \frac{1}{2}\left(x_{i}+x_{j}+x_{r}\right)^{2} \\
& +\sum_{i=1}^{m}\left(\frac{n_{i} \beta_{n_{i}}}{1-\beta_{n_{i}}}-\frac{n_{i}}{2}\right) x_{i}^{2}
\end{aligned}
$$

From eq. (3), it is easy to see that $\frac{n \beta_{n}}{1-\beta_{n}} \geq \frac{3}{5} n$ for $n \geq 3$. Hence, $f\left(x_{1}, x_{2}, \ldots, x_{m}\right)$ is positive for any none zero $\mathbf{X}$ and, consequently, $S$ is positive definite.

Based on the above lemma and theorem, it is easy to conclude that the iterative interpolation process for Loop subdivision is convergent.

Theorem 2 The iterative interpolation process for Loop subdivision surface is convergent.

Proof: The iterative process is convergent if and only if absolute value of the eigenvalues of the matrix $P=I-B$ are all less than 1 , or all eigenvalues $\lambda_{i}, 1 \leq i \leq m$, of $B$ are $0<\lambda_{i} \leq 1$.

Since $\|B\|_{\infty}=1$, we have $\lambda_{i} \leq 1$. On the other hand, since $B$ is the product of two positive definite matrices $D$ and $S$, following Lemma 1, all its eigenvalues must be positive. Hence, the iterative process is convergent.

\section{Extension to Open Meshes}

Loop subdivision surface based progressive interpolation technique can be used for open meshes as well. Actually the same advantages hold for open meshes too. Before we present Loop subdivision surface based progressive interpolation technique for open meshes, we need to review subdivision rules for the boundaries of an open mesh first.

Two kinds of boundary rules have been presented for Loop subdivision in the literature [19, 20, $21,22]$. In this paper, we follow the rules presented in [19, 20]. These rules, together with the Loop subdivision schemes, generate a smooth surface that is $C^{1}$ continuous at the boundaries [20, 19].

For these rules to work, the vertices on the boundary are divided into two categories: regular vertices and extraordinary vertices. A boundary vertex is called a regular vertex if its valence is 4 , as the one shown in Fig.1(a). Otherwise, a boundary vertex is called an extraordinary vertex.

For each existing boundary vertex, a new vertex is computed as a linear combination of the existing vertex and its two neighbors with weights $3 / 4,1 / 8$ and $1 / 8$, respectively. This vertex formula applies to both regular vertices and extraordinary vertices. 
For each boundary edge, a new edge vertex is generated in two ways. If the endpoints of the edge are both regular or both extraordinary, then the new vertex is just the average of the endpoints. If one of them is regular and the other one is extraordinary, then the new vertex is a linear combination of the regular vertex and the extraordinary vertex with weights $5 / 8$ and $3 / 8$, respectively, as in Fig.1(c).

Limit points are computed using two formulas, one for regular vertices and one for extraordinary vertices, as in Fig.1(e) and Fig.1(f). These formulas require both neighbors to be regular vertices. On boundaries of the initial mesh, one vertex could have either regular or extraordinary vertices. There are totally 6 different configurations. For each configuration, we can get a new limit point formula by combining the boundary subdivision rules with the standard limit points. These 6 limit point formulas are shown in Fig.1(e) to Fig.1(j).

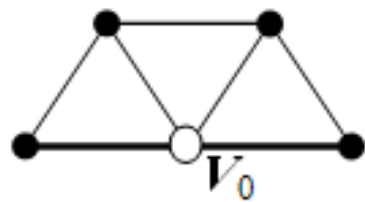

(a) Regular vertex

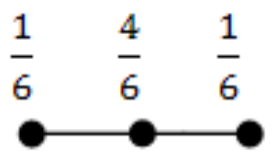

(e)

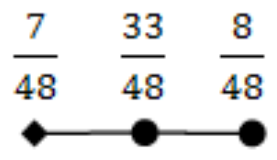

(h)

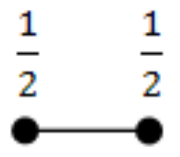

(b)

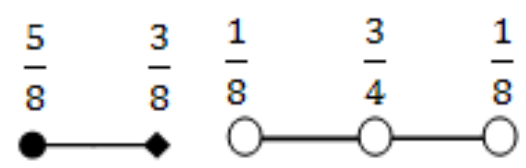

(c)

(d)

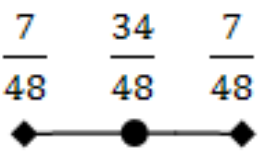

(g)

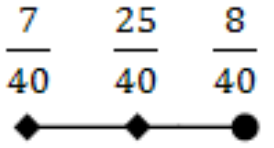

(j)

Figure 1: Boundary subdivision rules (b)-(d) and limit point forumlas (e)-(j), where the solid circular points are regular vertices and rectangle points are extraordinary vertices.

Note that boundary subdivision involves only the boundary vertices. Therefore interpolation can be performed for boundary vertices first. Once we have all the boundary vertices, interpolation of interior vertices is then performed without making any changes to the boundary vertices. The final mesh will interpolate both the boundary vertices and the interior vertices exactly. Interpolation of the boundary vertices is done using our progressive interpolation technique. The convergence of the interpolation is guaranteed. A mesh could have several disconnected closed boundaries, such as 6 in the pipe model in Fig.3(a). For each closed boundary, a linear system of equations can be built based on the limit point formulas for boundary vertices.

$$
\mathbf{V}_{\mathrm{B}}^{\infty}=\mathbf{E V}_{\mathrm{B}}
$$

Since very row is from one of the 6 limit point formulas, then $\mathbf{E}$ must be a strictly diagonally dominant matrix which means $\sum_{j=1, j \neq i}^{n} e_{i j}<e_{i i}$. The eigenvalues $\lambda_{i}$ of $\mathbf{E}$ satisfy $\left|\lambda_{i}\right| \leq 1$ for $\sum_{j=1}^{n} e_{i j}=1$. Thus the eigenvalues of $\mathbf{E}$ are in $(0,1]$. The advantage of our technique is very desirable. No matter how many disconnected boundaries there are, interpolation is done for all boundaries at the same time just through the local geometric operations. It avoids explicitly solving several linear equations separately.

Interpolation of interior vertices also uses the progressive interpolation technique. Its convergence is also provable. Let $\mathbf{V}=\mathbf{V}_{\mathrm{B}} \cup \mathbf{V}_{\mathrm{I}}$ be the vertex set of the initial mesh $M$, where $\mathbf{V}_{\mathrm{B}}$ and $\mathbf{V}_{\mathrm{I}}$ are the 
set of boundary vertices and interior vertices, respectively. If we add one extra vertex q to $M$ and connects every boundary vertices with $\mathbf{q}$, we get an closed mesh $M^{\prime}$ with vertex set $\mathbf{V}_{\mathrm{C}}=\mathbf{V} \cup \mathbf{q}$.

Applying the vertex limit point formula Eq.3 to interior vertices, we get a linear equation:

$$
\begin{aligned}
\mathbf{V}_{\mathrm{I}}^{\infty} & =\mathbf{W} \mathbf{V} \\
& =\mathbf{W}_{\mathrm{I}} \mathbf{V}_{\mathrm{I}}+\mathbf{W}_{\mathrm{B}} \mathbf{V}_{\mathrm{B}}
\end{aligned}
$$

$\mathbf{W}_{\mathrm{I}}$ is a submatrix of $\mathbf{W}$ consisting of $\left|\mathbf{V}_{\mathrm{I}}\right|$ columns of $\mathbf{W}$ corresponding to the interior vertices. $\mathbf{W}_{\mathrm{B}}$ is a similar submatrix corresponding to the boundary vertices. $\mathbf{W}_{\mathrm{I}}$ is similar to $\mathbf{B}$ for closed meshes. It can be decomposed into one diagonal matrix $\mathbf{D}_{\mathrm{I}}$ and a symmetric matrix $\mathbf{S}_{\mathrm{I}}$.

For this new closed mesh $M^{\prime}$, it is okay to apply the progressive interpolation technique developed in the previous sections. Therefore, the following equations hold.

$$
\mathbf{V}_{\mathrm{C}}^{\infty}=\mathbf{F V}_{\mathrm{C}}
$$

That is,

$$
\left[\begin{array}{c}
\mathbf{V}_{\mathrm{I}}^{\infty} \\
\mathbf{V}_{\mathrm{B}}^{\infty} \\
\mathbf{q}^{\infty}
\end{array}\right]=\left[\begin{array}{cc}
\mathbf{W}_{\mathrm{I}} & 0 \\
\mathbf{W}_{\mathrm{B}} & 0 \\
\mathbf{w}_{\mathrm{q}} & \alpha_{q}
\end{array}\right]\left[\begin{array}{c}
\mathbf{V}_{\mathrm{I}} \\
\mathbf{V}_{\mathrm{B}} \\
\mathbf{q}
\end{array}\right]
$$

It is clear that $\mathbf{W}$ is just a submatrix of $\mathbf{F} . \mathbf{F}$ is decomposed into $\mathbf{D}_{\mathrm{C}}$ and $\mathbf{S}_{\mathrm{C}} \cdot \mathbf{S}_{\mathrm{C}}$ depends only on the topology of the mesh. $M$ is part of $M^{\prime}$. Therefore, $\mathbf{S}_{\mathrm{I}}$ is just a minor of a positive definite matrix $\mathbf{S}_{\mathrm{C}}$. Now it is clear that $\mathbf{W}_{\mathrm{I}}$ satisfies the convergent condition for progressive interpolation. The examples in Fig.3 show interpolation results of open meshes.

\section{Results}

The progressive interpolation process is implemented for Loop subdivision surfaces on a Windows platform using OpenGL as the supporting graphics system. Quite a few cases have been tested. Some of the closed cases (a hog, a rabbit, a tiger, a statue, a boy, a turtle and a bird) are presented in Figure 2. All the data sets are normalized, so that the bounding box of each data set is a unit cube. For each closed case, the given mesh and the constructed interpolating Loop surface are shown. The sizes of the data meshes, numbers of iterations performed, maximum and average errors of these cases are collected in Table 1.

Open mesh examples are shown in Fig. 3. The performance is about the same as the closed mesh examples. For instance, for the face model (299 vertices) shown in Fig.3(c), it takes 10 iterations to reach an error of 0.000998516 for boundary vertex interpolation and also 10 iterations to reach an error of 0.000896328 for interior vertex interpolation by the new pogressive interpolation technique.

Table 1: Loop surface based progressive interpolation: test results.

\begin{tabular}{|l|r|c|c|l|}
\hline Model & \# of vertices & \# of iterations & Max Error & Ave Error \\
\hline Hog & 606 & 10 & 0.000870799 & 0.000175255 \\
\hline Rabbit & 453 & 13 & 0.000932857 & 0.000111197 \\
\hline Tiger & 956 & 9 & 0.000720453 & 0.00014148 \\
\hline Statue & 711 & 11 & 0.000890806 & 0.000109163 \\
\hline Boy & 17342 & 6 & 0.000913795 & 0.000095615 \\
\hline Turtle & 445 & 10 & 0.000955765 & 0.0001726 \\
\hline Bird & 1129 & 9 & 0.000766811 & 0.000088345 \\
\hline
\end{tabular}

From these results, it is easy to see that the progressive interpolation process is very efficient and can handle large meshes with ease. This is so because of the expotential convergence rate of the iterative process. Another point that can be made here is, although no fairness control factor is added in the progressive iterative interpolation, the results show that it can produce visually pleasing surface easily. 


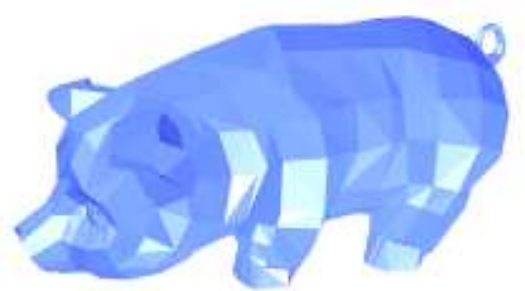

(a) Given mesh

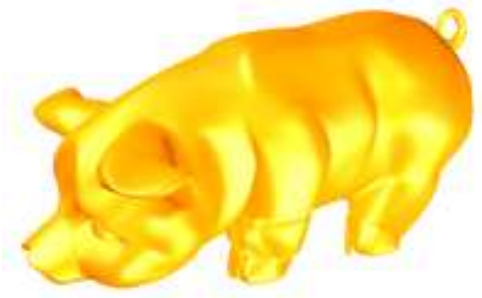

(b) I-surface

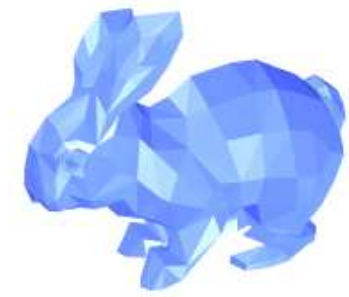

(c) Given mesh

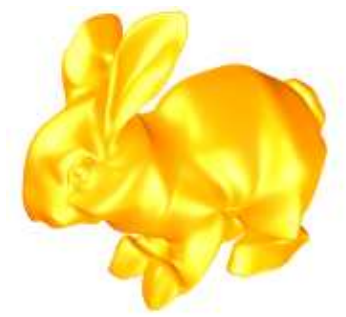

(d) I-surface

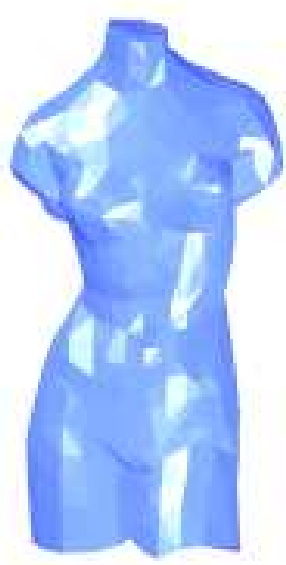

(g) Given mesh

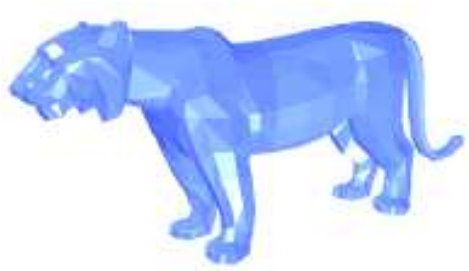

(e) Given mesh

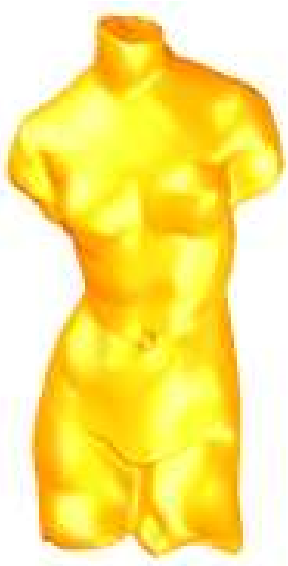

(h) I-surface

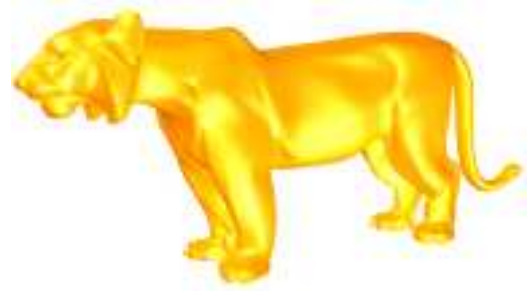

(f) I-surface

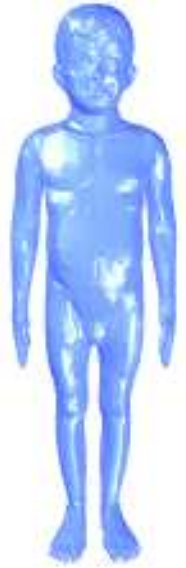

(i) G-mesh

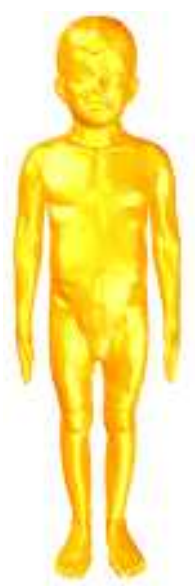

(j) I-surface

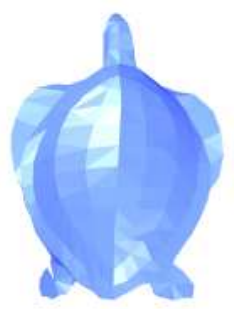

(k) Given mesh

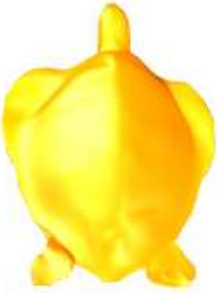

(l) I-surface

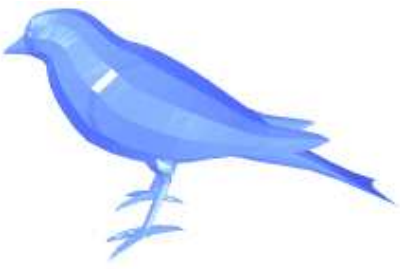

(m) Given mesh

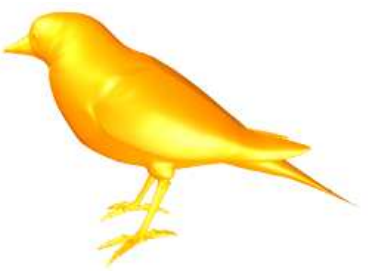

(n) I-surface

Figure 2: Examples of progressive interpolation using Loop subdivision surfaces (G-mesh $\equiv$ given mesh; I-surface $\equiv$ interpolating Loop surface). 


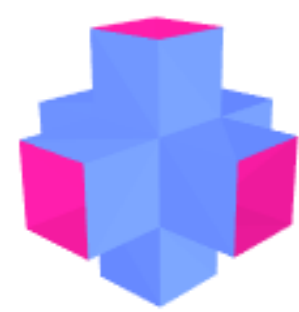

(a) Given mesh

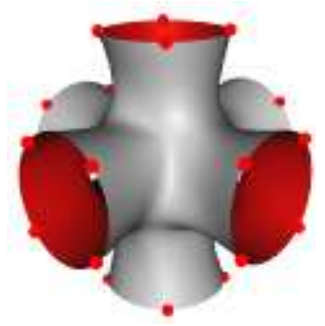

(b) I-surface

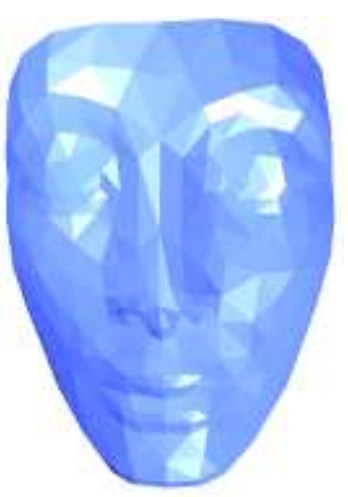

(c) Given mesh

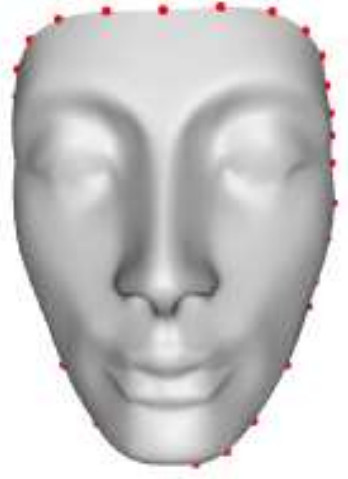

(d) I-surface

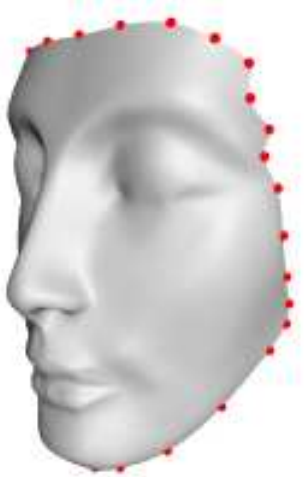

(e) Different view

Figure 3: Open mesh examples (I-surface $\equiv$ interpolating Loop surface). Red solid dots are boundary vertices of the given mesh.

\section{Concluding Remarks}

A progressive interpolation technique for Loop subdivision surfaces is presented and its convergence is proved. The limit of the iterative interpolation process has the form of a global method. Therefore, the new method enjoys the strength of a global method. On the other hand, since control points of the interpolating surface can be computed using a local approach, the new method also enjoys the strength of a local method. Consequently, we have a subdivision surface based interpolation technique that has the advantages of both a local method and a global method. The new technique works for both open and closed meshes. Our next job is to investigate progressive interpolation for Catmull-Clark and Doo-Sabin subdivision surfaces.

\section{Acknowledgement}

Research work presented here is supported by NSF (DMI-0422126). The last author is supported by NSFC(60625202, 60533070). Triangular meshes used in this paper are downloaded from the Princeton Shape Benchmark [17].

\section{References}

[1] Doo D, Sabin M, Behaviour of recursive division surfaces near extraordinary points, ComputerAided Design 1978, 10(6):356-360.

[2] Catmull E, Clark J, Recursively generated B-spline surfaces on arbitrary topological meshes, Computer-Aided Design 1978, 10(6):350-355.

[3] Halstead M, Kass M, DeRose T, Efficient, fair interpolation using Catmull-Clark surfaces, Proc.SIGGRAPH 1993, 47-61. 
[4] Nasri AH, Surface interpolation on irregular networks with normal conditions, Computer Aided Geometric Design 1991, 8:89-96.

[5] Zheng J, Cai YY, Interpolation over arbitrary topology meshes using a two-phase subdivision scheme, IEEE Trans. Visualization and Computer Graphics 2006, 12(3):301-310.

[6] Dyn N, Levin D, Gregory JA, A Butterfly Subdivision Scheme for Surface Interpolation with Tension Control, ACM Trans. Graphics 1990, 9(2):160-169.

[7] Litke N, Levin A, Schröder P, Fitting Subdivision Surfaces, Proc. Visualization 2001, 319-324.

[8] Loop C, Smooth Subdivision Surfaces Based on Triangles, Master'thesis, Dept. of Math., Univ. of Utah, 1987.

[9] Zorin D, Schroder P, Sweldens W, Interpolating Subdivision for Meshes with Arbitrary Topology, Computer Graphics, Ann. Conf. Series, 1996, 30:189-192.

[10] de Boor C, How does Agee's method work? Proc. 1979 Army Numerical Analysis and Computers Conference, ARO Report 79-3, Army Research Office, 299-302.

[11] Lin H, Bao H, Wang G, Totally positive bases and progressive iteration approximation, Computer \& Mathematics with Applications, 2005, 50:575-58.

[12] Lin H, Wang G, Dong C, Constructing iterative non-uniform B-spline curve and surface to fit data points, Science in China (Series E), 2003, 33:912-923 (in Chinese).

[13] Qi D, Tian,Z, Zhang Y, Zheng JB, The method of numeric polish in curve fitting, Acta Mathematica Sinica 1975, 18:173-184 (in Chinese).

[14] Delgado J, Peña JM, Progressive iterative approximation and bases with the fastest convergence rates, Computer Aided Geometric Design 2007, 24(1):10-18.

[15] Lai S, Cheng F, Similarity based Interpolation using Catmull-Clark Subdivision Surfaces, The Visual Computer 2006, 22(9):865-873.

[16] Magnus IR, Neudecker H, Matrix Differential Calculus with Applications in Statistics and Econometrics, New York, John Wiley \& Sons, 1988.

[17] Shilane P, Min P, Kazhdan M, Funkhouser T. The Princeton Shape Benchmark, Shape Modeling Int'l, 2004.

[18] Kobbelt L, Interpolatory Subdivision on Open Quadrilateral Nets with Arbitrary Topology, Comput. Graph. Forum 1996, 5(3):409-420.

[19] Hoppe H, DeRose T, Duchamp T, Halstead M, Jin H, McDonald J, Schweitzer J, Stuetzle W. Piecewise smooth surface reconstruction, SIGGRAPH '94: Proceedings of the 21st annual conference on Computer graphics and interactive techniques 1994, 295-302.

[20] Schweitzer J. E, Analysis and Application of Subdivision Surfaces, PhD thesis, University of Washington, Seattle, 1996.

[21] Zorin D, Schröder P, DeRose T, Kobbelt L, Levin A, Sweldens W. Subdivision for modeling and animation, SIGGRAPH 2000 Course Notes, ACM SIGGRAPH.

[22] Biermann H, Levin A, Zorin D. Piecewise smooth subdivision surfaces with normal control. Proceedings of SIGGRAPH 2000, 2000, 113-120. 